Cora-Lynn Munroe-Lynds

Dalhousie University, Halifax, Nova Scotia, Canada

Marc-André Simard

Université de Montréal, Montréal, Québec, Canada

Vinson Li

Dalhousie University, Halifax, Nova Scotia, Canada

Emily McClean

Dalhousie University, Halifax, Nova Scotia, Canada

Philippe Mongeon

Dalhousie University, Halifax, Nova Scotia, Canada

\title{
Mapping the Canadian library and information science research landscape (Paper)
}

\begin{abstract}
This work-in-progress paper aims to map the scholarship produced by the eight Canadian Library and Information Science (LIS) schools. After using the citation network to divide publications into several research areas, we analyze how the research output of different LIS schools is distributed across these areas, in an attempt to shed light on the schools' specificities and commonalities and how each school contributes to the global picture of Canadian LIS research.
\end{abstract}

\section{Introduction}

Information Science has been defined by Borko (1968) as "a discipline that investigates the properties and behaviour of information" concerned with the "origination, collection, organization, storage, retrieval, interpretation, transmission, transformation, and utilization of information". Because information is inherent to every discipline of research, Bates (1999) referred to information science as a meta-field. Empirical investigations of LIS have highlighted its multidisciplinary nature (Aharony, 2012; Chua \& Yang, 2008; Onyancha, 2018; Paul-Hus et al., 2016), as well as the gradual shift of the field's focus from libraries to a more diverse range of topics such as information technologies, knowledge management, and bibliometrics (Chua \& Yang, 2008; Figuerola et al., 2017; Larivière et al., 2012; Ma \& Lund, 2020; Onyancha, 2018). yet these previous studies all suffer from the same limitation: despite acknowledging the multidisciplinary nature of the field, they tend to ignore the differences in publication practices that characterize the disciplines composing the field and the potential biases that may result from these differences. For instance, they often use journal classifications to delineate the field, which 
can lead to both the inclusion of non-LIS articles published in multidisciplinary journals and the exclusion of LIS research published in non-LIS journals. Adopting an affiliation-based approach to mitigate these limitations, this work-in-progress delves into the composition of the Canadian LIS research landscape and highlights the specific role played by individual schools within it.

\section{Data and methods}

We collected the list of current Faculty members and Ph.D. students from the websites of the eight Canadian ALA-accredited LIS schools (American Library Association, 2020), and then retrieved their publications from the Web of Science. We used this first step to generate a list of department names (which are not controlled in the WoS and may thus vary), which we used to expand our dataset to include all articles published by previous faculty members or students that are no longer affiliated to the school and may thus not be listed on their website anymore. The resulting dataset is summarized in Table 1.

Table 1. Number of authors and publications identified for the eight Canadian LIS Schools

\begin{tabular}{lcc}
\hline Institution & Number of authors & Number of publications \\
\hline Dalhousie University (Dal) & 31 & 49 \\
McGill University (McGill) & 79 & 165 \\
University of Alberta (UofA) & 35 & 62 \\
University of British Columbia (UBC) & 43 & 60 \\
Université de Montréal (UdeM) & 46 & 132 \\
University of Ottawa (uOttawa) & 13 & 24 \\
University of Toronto (UofT) & 125 & 220 \\
University of Western Ontario (Western) & 105 & 258 \\
\hline All Universities & 404 & 941 \\
\hline
\end{tabular}

We mapped the Canadian LIS research landscape using the hybrid approach of Boyack and Klavans (2010), which combines bibliographic coupling and the words in the title and abstracts to calculate the weight of the network links. In our approach, we use TF-IDF to give more weight to rare words. In the resulting graph, each node represents a publication and belongs to one of twelve clusters identified by the Louvain algorithm (Bondel et al., 2008) with the resolution parameter set at 1.0 (Lambiotte et al., 2014) in Gephi. The labels of the clusters were chosen by ourselves, based on the titles of the publications in the clusters.

\section{Results}

The global map of Canadian LIS research is shown in figure 1. The four networks on the right are limited to publications of a specific LIS school. It indicates that the Canadian landscape of LIS research is the outcome of the schools' distinct research focus, as opposed to a uniform distribution of papers across research areas. 


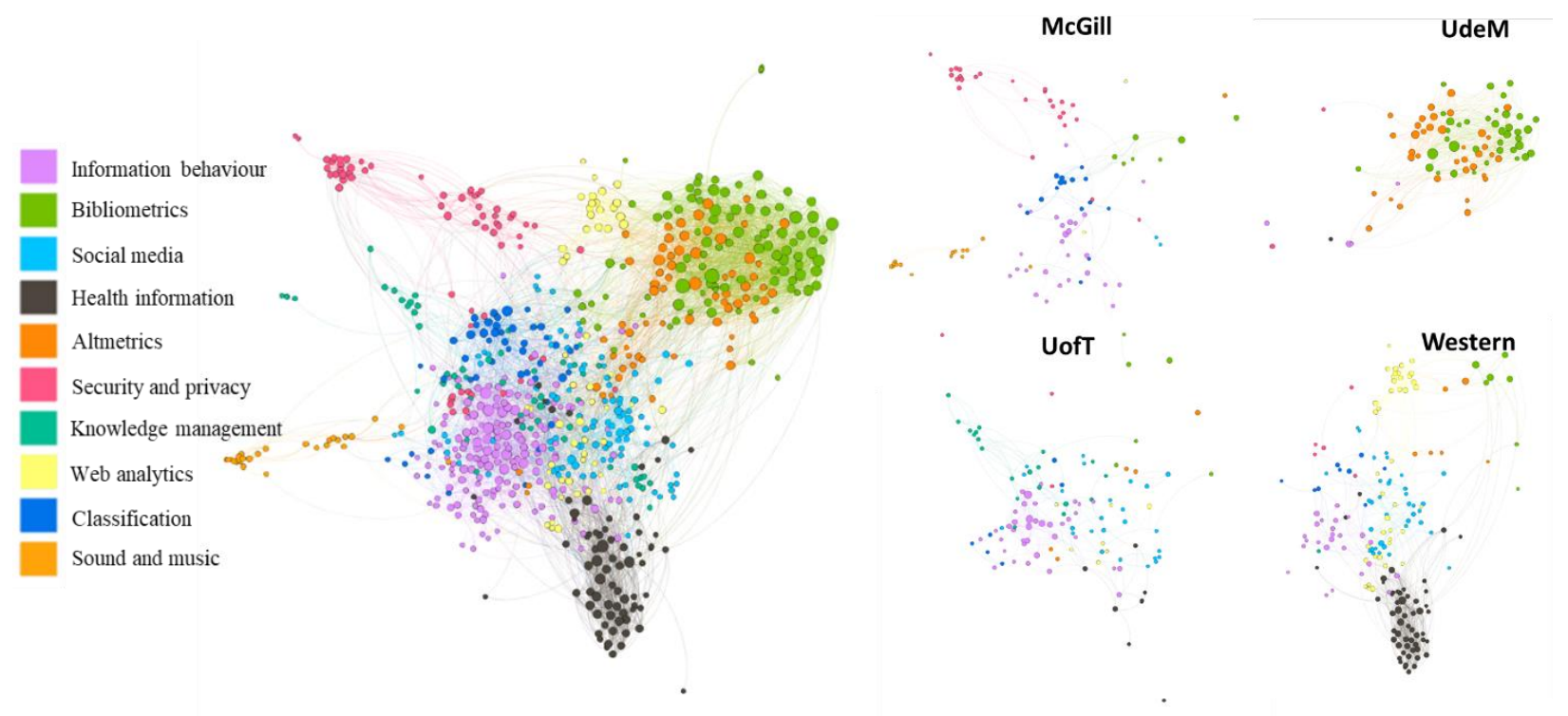

One issue with maps such as the ones presented in Figure 1 is that they do not take into account the different publication practices across research areas, giving more visibility to the (typically quantitative) areas where publication rates might be higher. Moreover, and especially when working with relatively small datasets as is the case here, these maps can be greatly influenced by the outputs of a few very prolific scholars. To mitigate this, we took individual researchers and calculated the relative frequency distribution of their research output across the research areas. This way, every researcher has an equal weight. Then, for each university, we calculated the relative frequency distribution of researchers across research areas, so that every school has the same weight. The average share of researchers in a research area thus provides a denominator to calculate the specialization index for each school-research area combination. A score below or above 1 respectively indicates a lower or higher than average number of researchers working in a specific area. Table 2 shows the respective areas of specialization of the eight Canadian LIS schools. We can see that the larger schools tend to cover a larger range of research, but still specialize in different areas. Smaller schools tend not to cover all the research areas, and thus tend to have higher specialization scores in the areas where they are active.

Table 2. Specialization of Canadian LIS departments based on researchers

\begin{tabular}{lcccccccc}
\hline Cluster & Dal & McGill & UofA & UBC & UdeM & UofO & UofT & Western \\
\hline Information behaviour & 0.85 & 1.17 & 1.64 & 1.05 & 0.68 & 1.10 & 1.17 & 0.72 \\
Classification & 2.03 & 1.20 & 1.29 & 0.68 & 1.27 & 0.00 & 0.71 & 0.94 \\
Bibliometrics & 0.51 & 1.53 & 0.65 & 0.36 & 2.00 & 2.99 & 0.48 & 0.32 \\
Altmetrics & 1.36 & 0.56 & 0.53 & 0.56 & 2.00 & 1.18 & 0.89 & 0.97 \\
Web and social media & 1.85 & 0.07 & 0.80 & 1.12 & 0.44 & 0.90 & 1.67 & 1.31 \\
Health information & 0.00 & 0.67 & 1.10 & 0.81 & 0.60 & 1.23 & 0.90 & 1.88 \\
Security and privacy & 0.00 & 1.89 & 0.00 & 1.99 & 1.15 & 0.00 & 0.87 & 0.86 \\
Knowledge management & 1.61 & 0.00 & 2.52 & 1.76 & 0.00 & 0.54 & 1.69 & 0.90 \\
Web analytics & 0.00 & 0.99 & 0.00 & 0.57 & 0.00 & 0.00 & 1.27 & 2.64 \\
Sound and music & 0.00 & 3.16 & 0.00 & 4.77 & 0.00 & 0.00 & 0.00 & 0.00 \\
\hline
\end{tabular}




\section{Conclusion}

Our results showed that the landscape in LIS research in Canada is built from the superposition of the schools' heterogenous research focuses rather than homogeneous distributions of research outputs across the different areas of research. This research in progress is currently limited to Web of Science data, which does not exhaustively cover the literature in the field (Meho \& Spurgin, 2005) may introduce some biases in the coverage (Mongeon \& Paul-Hus, 2016). The dataset is currently being expanded to include publications found in other databases and the researchers' CVs. Ultimately, our goal is to produce the first-ever comprehensive open dataset of Canadian LIS research publications, so that it can be used to better understand the field and its history.

\section{Reference List:}

Aharony, N. (2012). Library and Information Science research areas: A content analysis of articles from the top 10 journals $2007-8$. (1), https://doi.org/10.1177/0961000611424819

American Library Association. (2020). Database of ALA-Accredited Programs. Retrieved on July 28th 2020 from https://www.ala.org/CFApps/lisdir/index.cfm?

Bates, M. J. (1999). The invisible substrate of information science. Journal of the American Society for Information Science, 50(12), 1043-1050.

Bondel, V. D., Guillaume, J.-L., Lambiotte, R., \& Lefebvre, E. (2008). Fast unfolding of communities in large networks. Journal of Statistical Mechanics: Theory and Experiment, 2008(10), P10008-P10008.

Borko, H. (1968). Information science: What is it? American Documentation, 19(1), 3-5. https://doi.org/10.1002/asi.5090190103

Boyack, K. W., \& Klavans, R. (2010). Co-citation analysis, bibliographic coupling, and direct citation: Which citation approach represents the research front most accurately? Journal of the American Society for Information Science and Technology, 61(12), 2389-2404. https://doi.org/10.1002/asi.21419

Chua, A. Y. K., \& Yang, C. C. (2008). The shift towards multi-disciplinarity in information science. Journal of the American Society for Information Science and Technology, 59(13), 2156-2170. https://doi.org/10.1002/asi.20929

Figuerola, C. G., García Marco, F. J., \& Pinto, M. (2017). Mapping the evolution of library and information science (1978-2014) using topic modeling on LISA. Scientometrics, 112(3), 1507-1535. https://doi.org/10.1007/s11192-017-2432-9

Lambiotte, R., Delvenne, J.-C., \& Barahona, M. (2014). Laplacian Dynamics and Multiscale Modular Structure in Networks. IEEE Transactions on Network Science and Engineering, 1(2), 76-90. https://doi.org/10.1109/TNSE.2015.2391998 
Larivière, V., Sugimoto, C. R., \& Cronin, B. (2012). A bibliometric chronicling of library and information science's first hundred years. Journal of the American Society for Information Science and Technology, 63(5), 997-1016. https://doi.org/10.1002/asi.22645

Ma, J., \& Lund, B. (2020). The evolution of LIS research topics and methods from 2006 to 2018 : A content analysis. Proceedings of the Association for Information Science and Technology, 57(1). https://doi.org/10.1002/pra2.241

Meho, L. I., \& Spurgin, K. M. (2005). Ranking the research productivity of library and information science faculty and schools: An evaluation of data sources and research methods. Journal of the American Society for Information Science and Technology, 56(12), 1314-1331. https://doi.org/10.1002/asi.20227

Mongeon, P., \& Paul-Hus, A. (2016). The journal coverage of Web of Science and Scopus: A comparative analysis. Scientometrics, 106(1), 213-228. https://doi.org/10.1007/s11192$\underline{015-1765-5}$

Onyancha, O. B. (2018). Forty-Five Years of LIS Research Evolution, 1971-2015: An Informetrics Study of the Author-Supplied Keywords. Publishing Research Quarterly, 34(3), 456-470. https://doi.org/10.1007/s12109-018-9590-3

Paul-Hus, A., Mongeon, P., \& Shu, F. (2016). Portraying the Landscape of Canadian Library and Information Science Research = Portrait de la recherche en bibliothéconomie et sciences de l'information au Canada. Canadian Journal of Information and Library Science, 40(4), 332346. 